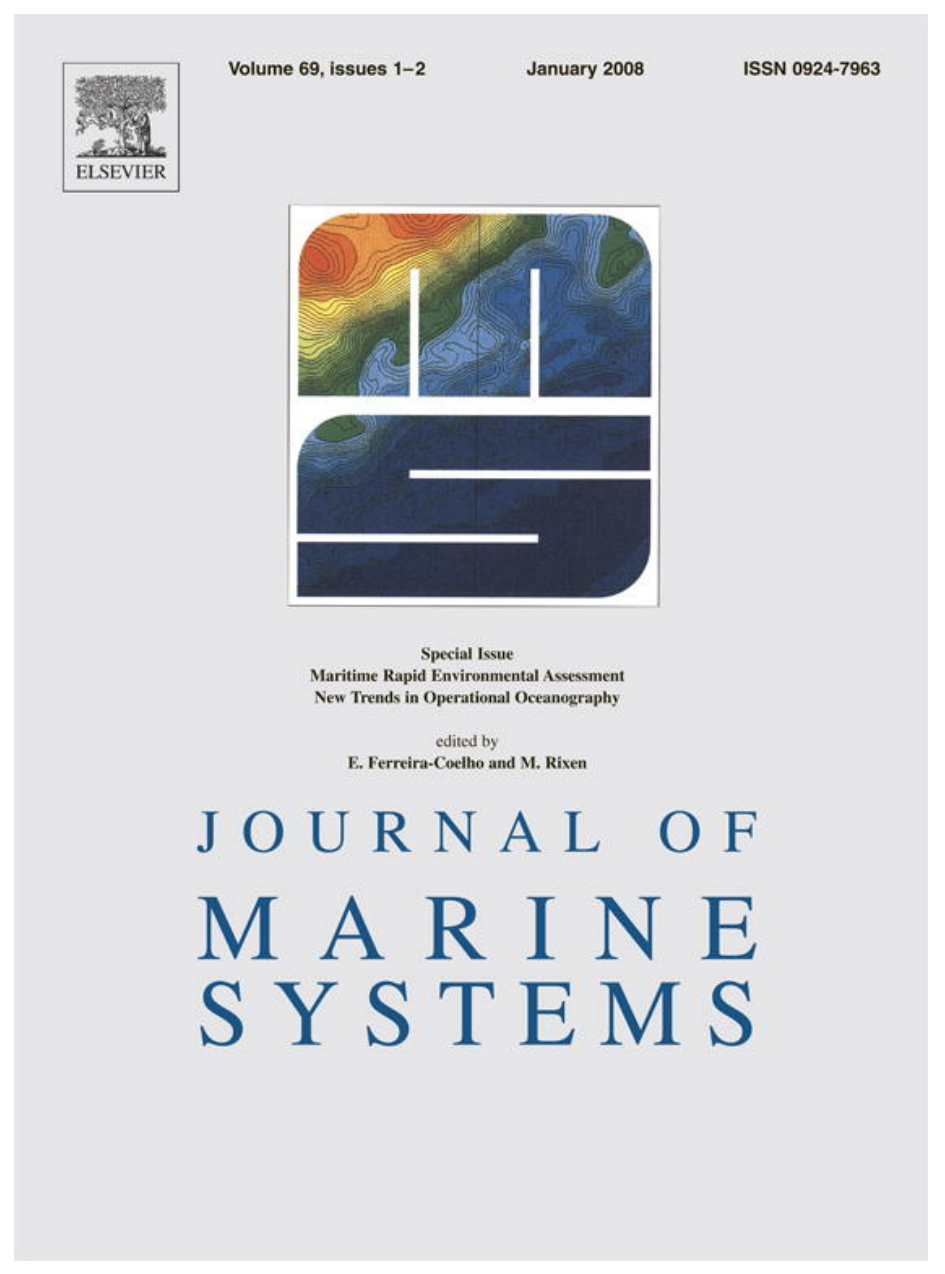

This article was published in an Elsevier journal. The attached copy

is furnished to the author for non-commercial research and education use, including for instruction at the author's institution, sharing with colleagues and providing to institution administration.

Other uses, including reproduction and distribution, or selling or licensing copies, or posting to personal, institutional or third party websites are prohibited.

In most cases authors are permitted to post their version of the article (e.g. in Word or Tex form) to their personal website or institutional repository. Authors requiring further information regarding Elsevier's archiving and manuscript policies are encouraged to visit: 


\title{
Environmental and acoustic assessment: The AOB concept ${ }^{\text {it }}$
}

\author{
N. Martins*, C. Soares, S. Jesus \\ Institute for Systems and Robotics, Universidade do Algarve, Campus de Gambelas, 8005-139 Faro, Portugal
}

Available online 7 February 2007

\begin{abstract}
The requirement for rapid environmental assessment has motivated the development of prediction tools, which allow the observation and prediction in very short notice, of the ocean evolution in an interval up to 3-4 weeks, in given littoral areas. Complex systems exist nowadays, where multidimensional quantities like the oceanographic-biogeochemical-optical-acoustic fields, are tracked in time, melding measures and models of some or all the involved quantities. At some point in the prediction system, the acoustic forecast is computed by acoustic propagation models taking as input the environmental forecast. Inevitably, the error of the acoustic forecast as given by the model output, originates from at least two error sources. The first is the environmental forecast error. The second is due to the model inaccuracies, and to the dependence of propagation on parameters not dealt with by the prediction system, like geometric or geo-acoustic properties. The acoustic community has developed a large number of acoustic inversion systems - based on e.g. matched-field processors or travel-time tomography - from which one can learn that an accurate acoustic simulation requires feeding the acoustic model with an environment which differs from the actual environment by a certain gap. This requires that the environmental forecast as given by ocean prediction systems be gap-compensated, prior to its inclusion in the acoustic environmental input. This paper puts the environmental gap in evidence, considering environmental forecasts, and historical and inverted data, to define heterogeneous environmental inputs to the propagation model. The corresponding acoustic outputs are compared to actual data from the MREA ' 03 sea trial. It is observed that acoustic inversion can play a significant role in converting the environmental forecast into the acoustic forecast.
\end{abstract}

(C) 2007 Elsevier B.V. All rights reserved.

PACS: $43.30 .+\mathrm{m}$

Keywords: Acoustic forecast; Matched-field processing; Tomography; Rapid environmental assessment

\section{Introduction}

Rapidly assessing the environmental conditions of a given coastal area, with the capability of being able to predict its evolution in the next days or weeks, has been

\footnotetext{
is This work was partially supported by FCT, under programs POSI and POCTI.

* Corresponding author.

E-mail addresses: nmartins@ualg.pt (N. Martins), csoares@ualg.pt (C. Soares), sjesus@ualg.pt (S. Jesus).

URL's: http://www.ualg.pt/siplab (N. Martins), http://www.ualg.pt/siplab (C. Soares), http://www.ualg.pt/siplab (S. Jesus)
}

the goal of many initiatives, since the end of the cold war, and the consequent shift of strategic regions to shallow areas. This requirement is triggered by naval needs, from which, the sonar performance (or acoustic detection probability) prediction is one of utmost relevance. In other words, it is important to predict the characteristics (amplitude or phase at frequencies of interest, time properties, etc.) of the acoustic signal received on a given data acquisition system, hence the concept of acoustic forecast. This purpose requires having at hand accurate oceanographic forecasts of e.g. the ocean temperature, salinity, currents, since these quantities directly influence acoustic propagation. Most efforts were carried out by 
oceanographic teams providing data of various natures (currents, sea surface temperature (SST), CTD, sea surface height (SSH), ADCP data, etc.) to accurate small-scale ocean observing and prediction systems (OOPSs), such as mini-HOPS, or the small-scale version of MODAS-NCOM (Martin, 2000). The OOPSs respond to operational assessment requests effectively on very short notice, providing nowadays forecast estimates of temperature, salinity, water currents, density, etc., typically up to one month (Robinson and Sellschopp, 2002). These systems work with interdisciplinary data and models, comprising ocean circulation models (in which, the oceanographic Primitive-Equation model plays a fundamental role), acoustic and biogeochemical data and models, assimilating in situ or remote sensed data, like SSH and SST, and modelling as well as tracking the forecast error (Robinson et al., 2002; Robinson and Lermusiaux, 2003). Testing has been going on for several years on the validation of such OOPSs in various scenarios. Among others, the guidelines for the OOPSs development are to decrease the error variance of various environmental parameter predictions, with a minimal model initialization.

The possibility of estimating future environmental conditions gave rise to operational applications for the navy, coupling the OOPSs with acoustic models, i.e., feeding the latter with the environmental forecasts. For tactical purposes, it was implicitly assumed that a decrease of the error variance of the environmental parameter predictions would necessarily imply a reduction of the variance of the acoustic detection probability prediction. This can be accepted as a general trend, but cannot be guaranteed. First, the data gridding is coarse in space-time, in oceanographic models, while it is fine in acoustic models (for example, internal tides, which have a significant acoustic impact, are not always taken into account in ocean circulation modelling (Lermusiaux and Chiu, 2002)). Second, the acoustic data model output accuracy is limited to a given frequency range and to the degree of knowledge about the environment. Third, the acoustic propagation model is parameterized by quantities that are not dealt with by the oceanographic model, e.g. bottom attenuation, density and thickness of different layers. Recent improvements in the OOPSs' structure have opened the possibility of obtaining more accurate acoustic forecasts, by assimilating ocean acoustic models and data in the ocean observing and prediction system, as is the case in HOPS (Robinson et al., 2002; Lermusiaux and Chiu, 2002). Here, acoustic quantities are incorporated in an interdisciplinary physical-acoustic state vector, which reflects the physical-acoustic link through the sound speed. Then, a physical-acoustic forecast error is tracked and minimized. Nevertheless, the eventual acoustic model environmental input error is modeled by a stochastic extension of the Primitive-Equation model, representing uncertainties due to sub-mesoscales and internal tides not accounted for in the deterministic mesoscale simulations (Lermusiaux and Chiu, 2002).

In the context of associating a given actual acoustic signal with an optimum acoustic model environmental input, the appearance of matched-field tomography (MFT) in the late 1980s, and its application to shallow water scenarios on the early 1990s, has opened at least two directions: first, the usage of acoustics for environmental purposes; second, it has given some insight into the sensitivity of the acoustic signal to environmental variations. A large number of experimental results obtained by various teams using MFT in shallow water regions, have shown the influence of internal tides and waves, the strong influence of bottom properties on signal propagation, and the crucial role of bathymetry and source-receiver geometry on the inversion process. Since even the most sophisticated propagation models existing today (and presumably in the years to come) cannot take into account all the real world details, in many situations the outcome of the inversion process that most closely matches the acoustic signal is an environment often "slightly shifted" from the actual measured environment, thus the concept of equivalent acoustic model. Hence, when the purpose is to predict the acoustic signal, even with an environmental forecast null error, the forecast should be modified in order to compensate the environmental model shift, to obtain the acoustic signal that is closest to the actual acoustic signal.

At this point, an acceptable goal would be to use the equivalent acoustic model computed at present time, with the oceanographic model predictions, to obtain accurate predictions of the acoustic field at some future time. In principle, a set of inversion outcomes and nowcasts could be used to 'fine tune' the environmental model to be combined with the oceanographic forecast, and finally compute the acoustic forecast. The bottom properties considered in the acoustic forecast would be strongly correlated with those estimated by acoustic inversion at present time, for geo-acoustic range-independent scenarios. As a matter of fact, the problem is not so simple, since the environmental shift of the inverted data may not be solely due to acoustic modelling issues, but also to errors in the inversion process, and noise implicitly leading to random environmental estimates effects often difficult to separate.

Using the acoustic and environmental data gathered during the MREA '03 sea trial, this paper intends to put 
in evidence the differences between the measured, forecast and inverted environmental quantities - mainly the water column temperature profiles - and the implications of these differences on modelling the acoustic data. Also, the Acoustic Oceanographic Buoy (Jesus et al., 2003) is presented as a promising tool for operational acoustic rapid environmental assessment (REA) of small coastal areas. This paper is structured as follows. Section 2 defines the problem of acoustic forecast, and summarily describes the Acoustic Oceanographic Buoy, Section 3 compares actual data with signal simulations with heterogeneous environmental models, and some conclusions are drawn in Section 4.

\section{Background}

\subsection{The acoustic forecast problem}

The problem of acoustic forecast can be defined as follows: given a set of acoustic and oceanographic models and quantities measured in a given area, till present time $t_{0}$, it is required to estimate the acoustic field in the same area, at a future time $t_{1}$. The problem takes place in an acoustic-oceanographic (AO) scenario. The actual oceanographic, geometric and geo-acoustic parameters are grouped into a time $(t)$-dependent vector, defining the ocean $\mathrm{AO}$ conditions:

$$
\begin{aligned}
\boldsymbol{\mu}(t) & =\left[T(t) S(t) H_{0}(t) \mathbf{s}^{\mathrm{T}}(t) \mathbf{h}^{\mathrm{T}}(t) \mathbf{b}^{\mathrm{T}}(t) \mathbf{I}^{\mathrm{T}}(t)\right]^{\mathrm{T}} \\
& =\left[\boldsymbol{\gamma}^{\mathrm{T}}(t) \boldsymbol{\beta}^{\mathrm{T}}(t)\right]^{\mathrm{T}},
\end{aligned}
$$

where $T(t), S(t), H_{0}(t), \mathbf{s}(t), \mathbf{h}(t)$ and $\mathbf{b}(t)$ designate the actual water column temperature, salinity and depth, the source and hydrophone set coordinates, and the geoacoustic parameters, respectively. The vector $\mathbf{l}(t)$ contains parameters that either do not influence significantly the acoustic field at the considered space-time scales, or are not always known with sufficient accuracy, like shear speeds, sea surface/bottom roughness, etc.. Due to the bidisciplinary nature of the problem at hand, the parameter set can be split into 2 sub-sets, namely the water column parameters $\boldsymbol{\gamma}(t)$ and the remaining parameters $\boldsymbol{\beta}(t)$. Considering an acoustic transmission through the medium described by these conditions, the acoustic receiving device (e.g. the AOB1, described in Section 2.3) will receive the actual acoustic signal spatially sampled by a set of $L$ hydrophones:

$$
\begin{aligned}
\mathbf{X}(t) & =g[\boldsymbol{\mu}(t)] \\
& =\left[\mathbf{Y}_{n}^{\mathrm{T}}\left(\boldsymbol{\mu}, f_{1}, t\right) \mathbf{Y}_{n}^{\mathrm{T}}\left(\boldsymbol{\mu}, f_{2}, t\right), \ldots, \mathbf{Y}_{n}^{\mathrm{T}}\left(\boldsymbol{\mu}, f_{K}, t\right)\right]^{\mathrm{T}},
\end{aligned}
$$

where $g$ stands for the physical transformation of the AO conditions into acoustics, and each $k$ th frequency-dependent vector $\mathbf{Y}_{n}\left(\boldsymbol{\mu}, f_{k}, t\right), k=1, \ldots, K$ is the $n$th snapshot of a single-frequency component of the signal received on the $L$ hydrophones. Note that X(t) contains the spectra of the signals received on a given time interval. Let us refer to Fig. 1, which illustrates a conceptual procedure to compute the acoustic forecast. By one side, we have at hand oceanographic and acoustic data, $\boldsymbol{\mu}\left(t_{0}\right)$ and $\mathbf{X}\left(t_{0}\right)$, respectively, at $t_{0}$. Making use of acoustic inversion, the acoustic data are transformed into the acoustic model input $\hat{\boldsymbol{\mu}}_{a}\left(t_{0}\right)$. Hereafter, the subscript ${ }_{a}$ will indicate that a given quantity is an estimate, by acoustic inversion, of the corresponding actual quantity in Eq. (1). The input $\hat{\boldsymbol{\mu}}_{a}\left(t_{0}\right)$

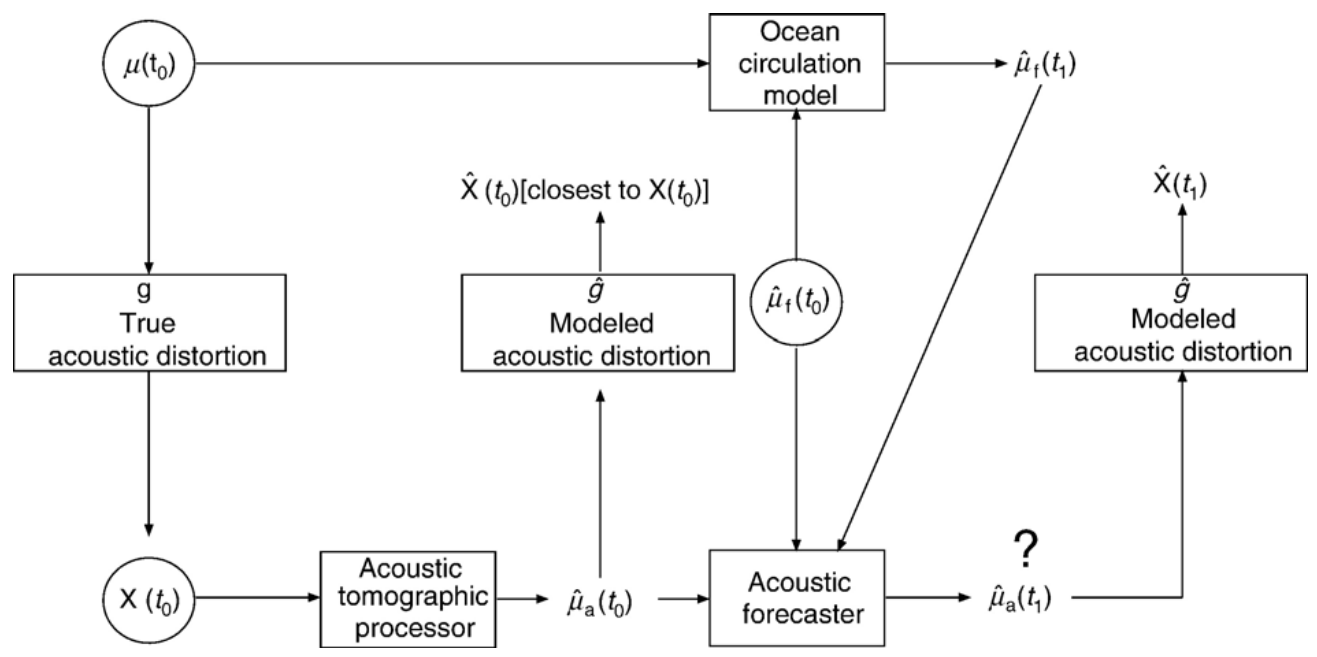

Fig. 1. Acoustic forecast as an acoustic model input parameter estimation problem. The AO quantities available at $t_{0}$ are enclosed by circles: the measured oceanographic data $\boldsymbol{\mu}\left(t_{0}\right)$, measured acoustic data $\mathbf{X}\left(t_{0}\right)$ and oceanographic nowcast $\hat{\boldsymbol{\mu}}_{f}\left(t_{0}\right)$. The acoustic model input at $t_{0}$, the nowcast and the forecast serve as input to the acoustic forecaster, to estimate the acoustic model input at $t_{1}$. 
is the vector corresponding to the simulated signal closest to the data $\mathbf{X}\left(t_{0}\right)$. By the other side, we have at hand the forecast of the physical conditions, $\hat{\boldsymbol{\mu}}_{f}\left(t_{1}\right)=$ $\left[\hat{\boldsymbol{\gamma}}_{f}^{\mathrm{T}}\left(t_{1}\right) \hat{\boldsymbol{\beta}}_{f}^{\mathrm{T}}\left(t_{1}\right)\right]^{\mathrm{T}}$. The point now is to associate the forecast $\hat{\boldsymbol{\mu}}_{f}\left(t_{1}\right)$ with an optimum simulated acoustic signal $\hat{\mathbf{X}}\left(t_{1}\right)$. The acoustic model parameter that generates $\hat{\mathbf{X}}\left(t_{1}\right)$ is structured as $\hat{\boldsymbol{\mu}}_{a}\left(t_{1}\right)=\left[\hat{\boldsymbol{\gamma}}_{a}^{\mathrm{T}}\left(t_{1}\right) \hat{\boldsymbol{\beta}}_{a}^{\mathrm{T}}\left(t_{1}\right)\right]^{\mathrm{T}}$. The determination of the optimum values for the parameters in $\hat{\boldsymbol{\mu}}_{a}\left(t_{1}\right)$ is equivalent to ideally solving the acoustic forecast problem:

$$
\left\{\begin{array}{l}
\hat{\mathbf{X}}\left(t_{1}\right)=\hat{g}\left[\hat{\boldsymbol{\mu}}_{a}\left(t_{1}\right)\right] \\
\hat{\boldsymbol{\mu}}_{a}\left(t_{1}\right)=\arg \min _{\boldsymbol{\mu}_{a s}\left(t_{1}\right)} U\left[\mathbf{X}\left(t_{1}\right), \hat{g}\left(\boldsymbol{\mu}_{a s}\left(t_{1}\right)\right)\right],
\end{array}\right.
$$

where $U\left[\mathbf{X}\left(t_{1}\right), \hat{g}\left(\boldsymbol{\mu}_{a s}\left(t_{1}\right)\right)\right]$ is a cost function which measures the proximity between the actual and simulated signals. The vector $\boldsymbol{\mu}_{a s}\left(t_{1}\right)$ is a generic AO candidate, and $\hat{g}$ stands for the model of the actual acoustic distortion. The acoustic model output is the acoustic signal forecast $\hat{\mathbf{X}}\left(t_{1}\right)$. The acoustic forecast can be constrained to lie in a given subspace, if there is a priori knowledge about the physics. Note that it is impossible to carry out the minimization in Eq. (3), since the future acoustic signal $\mathbf{X}\left(t_{1}\right)$ is unknown. Even though, having at hand acoustic data gathered at sea, at present time $-\mathbf{X}\left(t_{0}\right)$ - it is possible to 'learn' the relationship between the nowcast $\hat{\boldsymbol{\mu}}_{f}\left(t_{0}\right)$ and the structure of $\hat{\boldsymbol{\mu}}_{a}\left(t_{0}\right)$. Then, this relationship can be transposed to the future, to transform $\hat{\boldsymbol{\mu}}_{f}\left(t_{1}\right)$ into the acoustic forecast $\hat{\mathbf{X}}\left(t_{1}\right)$. This is represented in Fig. 1, by feeding the "acoustic forecaster" with the nowcast $\hat{\boldsymbol{\mu}}_{f}\left(t_{0}\right)$, the AO inversion result $\hat{\boldsymbol{\mu}}_{a}\left(t_{0}\right)$ and the forecast $\hat{\boldsymbol{\mu}}_{f}\left(t_{1}\right)$.

Note that every component of $\hat{\boldsymbol{\mu}}_{a}\left(t_{1}\right)$ is a variable which contributes to maximize the 'acoustic proximity'. Since the proximity between the actual and every candidate acoustic signal is a function of the entire vector $\boldsymbol{\mu}_{a s}\left(t_{1}\right)$, it may happen that e.g. $\hat{T}_{a}\left(t_{1}\right)$ or $\hat{S}_{a}\left(t_{1}\right)$ differ respectively from $T\left(t_{1}\right)$ and $S\left(t_{1}\right)$, or from $\hat{T}_{f}\left(t_{1}\right)$ and $\hat{S}_{f}\left(t_{1}\right)$. This is a consequence of the inaccuracies of: the inversion method (data model, a priori assumptions, etc.) and the acoustic model input parameterization (for instance, when all inhomogeneities of the physical medium cannot be taken into account). Also, the acoustic model response is non-monotonic in the environmental input. The combination of all these factors implies a non-monotonic relationship between 'oceanographic proximity' and 'acoustic proximity'. While the oceanographic forecast is the result of the minimization of functions involving observed and modeled oceanographic states, the outlined acoustic forecast is intended to minimize the distance between observed and modeled acoustic signals derived from those oceanographic states. Regarding the environmental parameterization of the acoustic model, in principle, the parameter vector should include acoustically-influent parameters, at the forecast frequencies. For example, a very inaccurate acoustic forecast is expected at high frequencies, if the bottom parameters are inverted with low frequencies.

\subsection{Acoustic model input estimation by matched-field acoustic inversion}

The determination of the acoustic propagation model input parameter that maximizes the 'acoustic proximity' is explained in this section. It constitutes a synchronous estimation, since, for a given signal $\mathbf{X}(t)$, it gives as output the vector $\hat{\boldsymbol{\mu}}_{a}(t)$, containing the environment estimate. The underlying method falls into the category of matched-field processing, well known as a means of estimating the physical state that constrains acoustic propagation giving rise to the received acoustic signal. The observed signal is inverted to the physical state.

The assumed data model decomposes Eq. (2) as

$\mathbf{X}(\boldsymbol{\mu})=\mathbf{H}(\boldsymbol{\mu}) \mathbf{S}+\mathbf{U}$,

where $\mathbf{H}(\boldsymbol{\mu})$ is a broadband channel transfer function matrix, structured as

$\mathbf{H}=\left[\begin{array}{cccc}\underline{H}\left(f_{1}, \boldsymbol{\mu}\right) & \mathbf{0} & \cdots & \mathbf{0} \\ \mathbf{0} & \underline{H}\left(f_{2}, \boldsymbol{\mu}\right) & \cdots & \mathbf{0} \\ \vdots & \vdots & \ddots & \vdots \\ \mathbf{0} & \mathbf{0} & \cdots & \underline{H}\left(f_{K}, \boldsymbol{\mu}\right)\end{array}\right]$

where $\underline{H}\left(f_{k}, \boldsymbol{\theta}\right)$ is a vector with the (narrowband) channel transfer function at $k$ th frequency, assumed time invariant during the temporal observation window, and $\mathbf{U}$ is an additive noise term, assumed zero mean and white. The vector $\mathbf{S}$ accounts for the source signal and random acoustic distortions not included in $\mathbf{H}(\boldsymbol{\mu})$. The (normalized) incoherent processor is given by

$P(\boldsymbol{\mu})=\frac{\sum_{\mathrm{k}=1}^{\mathrm{K}} \underline{H}^{\mathrm{H}}\left(f_{k}, \boldsymbol{\mu}\right) \hat{\mathbf{C}}_{Y Y}\left(f_{k}, \boldsymbol{\mu}\right) \underline{H}\left(f_{k}, \boldsymbol{\mu}\right) / \hat{\lambda}_{\max , k}}{\sum_{\mathrm{k}=1}^{\mathrm{K}}\left\|\underline{H}\left(f_{k}, \boldsymbol{\mu}\right)\right\|^{2}}$,

where $\hat{\mathbf{C}}_{Y Y}\left(f_{k}, \boldsymbol{\mu}\right)=\sum_{n=1}^{N} \mathbf{Y}_{n}\left(f_{k}, \boldsymbol{\mu}\right) \mathbf{Y}_{n}^{H}\left(f_{k}, \boldsymbol{\mu}\right) /\left[N \| \mathbf{Y}_{n}\left(f_{k}\right.\right.$, $\left.\boldsymbol{\mu}) \|^{2}\right]$ is the sample correlation matrix computed from $N$ snapshots, and $\hat{\lambda}_{\text {max }, k}$ is the estimate of the maximum eigenvalue of $\mathbf{C}_{Y Y}\left(f_{k}, \boldsymbol{\mu}\right)$. This processor is a particular form of the broadband processor proposed in Soares and Jesus (2003), where here it is assumed that the source power spectrum is unknown and flat.

\subsection{The Acoustic Oceanographic Buoy}

For the purpose of acoustic REA, an acquisition system was developed to measure and estimate the 
temperature and acoustic fields, in the operational area of interest. This system is the Acoustic Oceanographic Buoy-version 1 (AOB1), which is a light $(\approx 80 \mathrm{~kg}$, including all sensors and batteries) receiving device which incorporates last generation technology for acquiring, storing and processing acoustic, positioning and temperature signals received on four vertically separated sensors. The physical characteristics of the AOB1, in terms of size, weight and autonomy, will tend to those of a standard sonobuoy, with however the capability of local data storage, processing and online transmission. Data transmission is ensured by seamless integration into a wireless local area network, which allows for space-variant tomography within ranges up to $20 \mathrm{~km}$. The system bandwidth reaches $15 \mathrm{kHz}$, which allows its usage in other applications, such as active sonar and underwater communications.

The AOB1 hardware is described, in more detail, in Jesus et al. (2003) and Soares et al. (2004). The AOB1 software has the function of online controlling, monitoring and inverting the collected data. In this preliminary test, the software was separated into two parts: the buoy control and monitoring, and the online data inversion. The buoy control and monitoring was performed by a specially developed Windows operating system oriented program running on a laptop. This computer was fitted with a wireless pc-card attached to an omnidirectional $12 \mathrm{dBi}$ outdoor antenna via a $1 \mathrm{~W}$ amplifier. It was also possible to transfer acoustic data from the AOB1 via ftp, for on-board online inversion. The inversion software was based on code previously developed for blind ocean acoustic tomography (Jesus et al., 2006), with different settings for the parameter search bounds and the genetic algorithm conversion parameters, taking into account that the source position was approximately known, and that only very few hydrophones were available.

\section{Data/model comparison}

Environmental assessment results are presented in this section, where the inverted oceanographic field is compared to the ground truth field observed on the MREA '03 sea trial. The purpose of this comparison is to focus on the importance of acoustic inversion in minimizing the variance of the sonar performance prediction. It addresses general issues to be taken into account, when defining an estimator of $\hat{\boldsymbol{\mu}}_{a}\left(t_{1}\right)$. Although a closed-form approach to the estimation of $\hat{\boldsymbol{\mu}}_{a}\left(t_{1}\right)$ is not presented, it is discussed the impact of diverse combinations of predicted, inverted and measured oceanographic, geometric and geo-acoustic data - which define $\hat{\boldsymbol{\mu}}_{a}\left(t_{1}\right)$ - on the prediction of the acoustic field. It is expected that the optimum vector $\hat{\boldsymbol{\mu}}_{a}\left(t_{1}\right)$ be a function of some of those quantities.

(a)
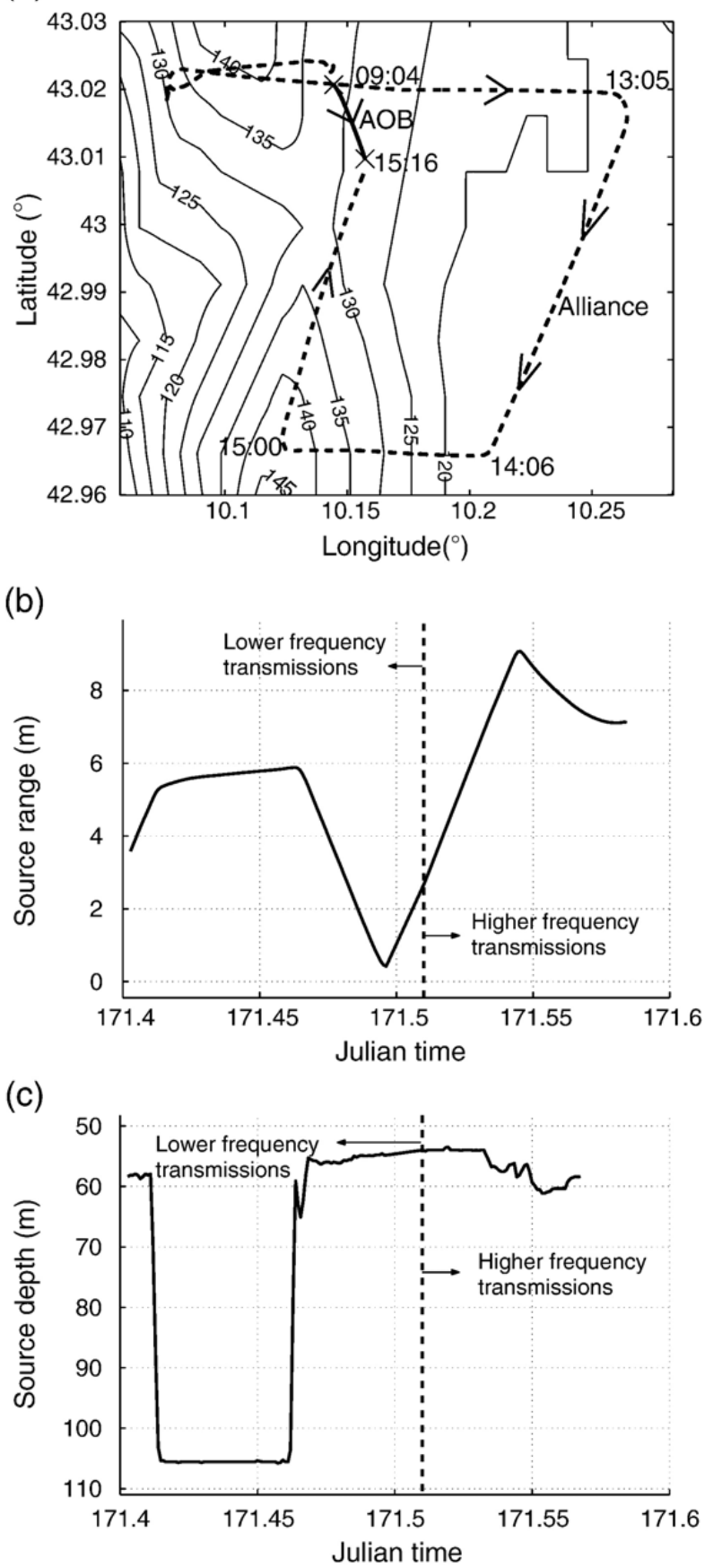

Fig. 2. Source-AOB1 geometry, estimated from GPS recordings. (a) Source and AOB1 tracks, superimposed to the bathymetry in contour lines. The acoustic source was carried by the R/V Alliance, defining the trajectory in the dashed line. The AOB1 was free drifting - continuous line - during the whole acoustic trial, from 09:04 to 15:16 June 21st. (b) Time-variant source range and (c) depth. The acoustic trial has comprised frequency transmissions on the bands $500-800$ and $900-1200 \mathrm{~Hz}$. 
(a)

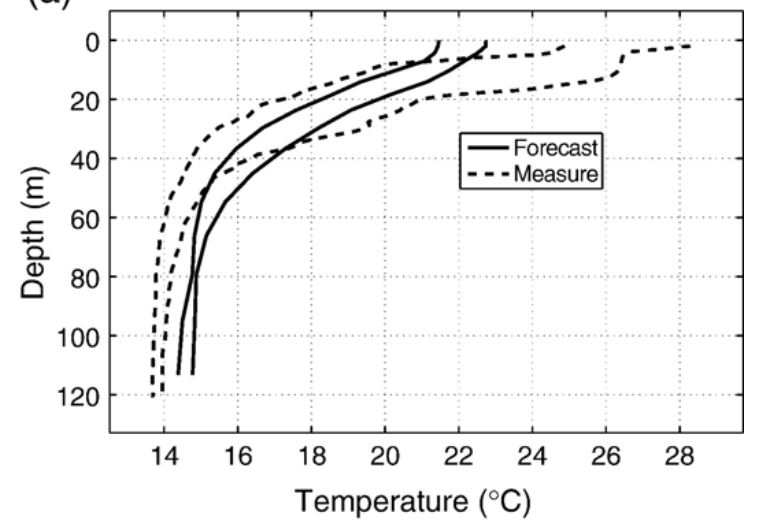

(b)

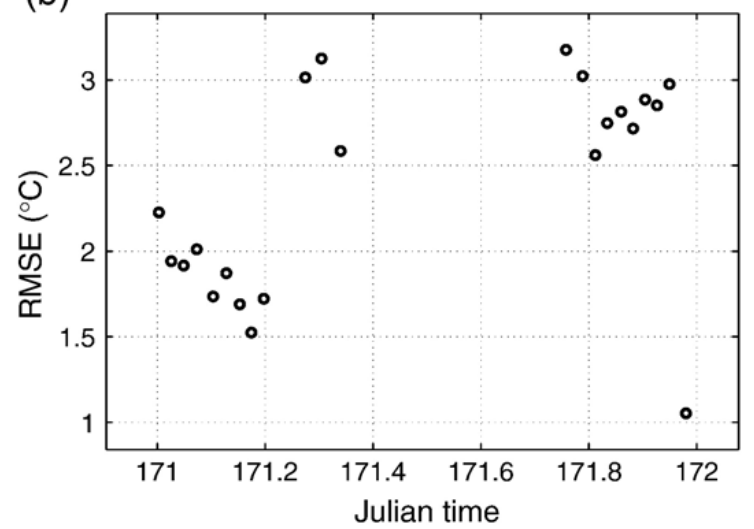

Fig. 3. (a) Temperature forecast bounds (solid lines) overimposed to the CTD measure bounds (dashed lines) in the MREA '03 sea trial, for June 21 st. The forecast bounds are those for the time instants closest to the CTD cast times. (b) Depth-averaged root-mean-square-error (RMSE) of the forecasts, along time.

\subsection{The MREA '03 sea trial and oceanographic forecasts for the trial area}

The MREA '03 sea trial took place off the west coast of Italy, in the north Elba Island area, during June 2003, and involved a bi-disciplinary team working on the acoustic and oceanographic fields. The sea trial comprised a set of acoustic and oceanographic measurements, to be combined with models, with the goal of REA. The oceanographic instrumentation consisted of ADCPs, current-thermistor chains, one meteo buoy, one CTD, Lagrangian drifters and one directional wave rider buoy, between other instrumentation. The acoustic component of the campaign involved an acoustic source, deployed from the $\mathrm{R} / \mathrm{V}$ Alliance at a variable depth between 60 and $100 \mathrm{~m}$ (depending on the ship speed) and the recently developed Acoustic-Oceanographic Buoy as the acoustic acquisition system, deployed on June 21st, with very favorable weather conditions, in an area of mild range-dependency. The buoy was deployed at Julian time (JT) 171.412, and recovered at JT 171.719. Fig. 2 shows the source-receiver geometry, estimated from global positioning system (GPS) recordings. The source-receiver range varied between 0.5 and $9 \mathrm{~km}$. The bathymetric signature varies between 115 and $145 \mathrm{~m}$ on the acoustic trial area. The data acquisition objective was twofold: (1) to permit source localization, using a single buoy with a few hydrophones in an unknown and range-dependent environment, and (2) to perform tomographic inversions for the environmental parameters, which serve as input to maritime REA purposes. Broadband 2-s duration linear frequency modulation (LFM) signals were transmitted in a lower and a higher band, 500-800 Hz and 900-1200 Hz, respectively, in JT intervals 171.403-171.496 and 171.510171.584 , respectively. The repetition rate was $8 \mathrm{~s}$.

A conventional approach to REA was implemented, combining measures of the ocean conditions with an ocean circulation model, to obtain estimates of the threedimensional water column temperature and salinity. The considered model, tailored for the regional area of interest, is the data-assimilative re-locatable model MODAS-NCOM (Martin, 2000). This model took as input SSH and SST satellite measures, as well as archival water column temperature profiles in the area. For June 21 st, a nowcast and a set of 8 forecasts were calculated at $0 \mathrm{~h}$ of the day, for the whole day, with a 3-h interval. Also, a set of 22 CTD measurements was taken in the area, every $67 \mathrm{~min}$ in average, spanning the whole day. Fig. 3 shows the temperature forecasts and the measured temperature profiles. The high values for the depthaveraged RMSE between the actual and estimated profiles, in Fig. 3(b), can be explained by the fact that the oceanographic model assimilates only SSH and SST



Fig. 4. Baseline model for the MREA '03 acoustic data inversions. All model parameters are range-independent, except the water depth. 
Table 1

Genetic algorithm settings for environmental assessment

\begin{tabular}{lc}
\hline Parameter & Setting \\
\hline Generations & 30 \\
Population size & 200 \\
Independent populations & 1 \\
Mutation probability & 0.004 \\
Crossover probability & 0.9 \\
Number of crossover points & 4 \\
\hline
\end{tabular}

measures. Even though, the water surface temperature forecast differs significantly from the measured values, possibly due to the presence of clouds in some of the satellites vision fields. The temperature and salinity forecasts (the latter not shown here) define the vector $\boldsymbol{\mu}_{f}(t)$, for all the 8 forecast outcomes for June 21 st.

\subsection{Environmental assessment results}

Given the acoustic data received on the AOB1 on June 21st, a set of tomographic inversions was carried out. In particular, two empirical orthogonal functions of temperature were considered. Their coefficients search bounds were set according to the temperature profile time-variability observed on CTD measurements along days 16, 17 and 19 of June. The baseline environmental model is shown in Fig. 4, indicating 3 out of 4 AOB1 hydrophones that were processed, thus selected due to the presence of strong impulsive noise on the 4th hydrophone. The baseline parameter values were taken from Gingras (1994). The considered forward model was the normal mode propagation model C-SNAP (Ferla et al., 1993).

Considering that the source position history is known see Fig. 2 - the water column temperature and all the bottom parameters in Fig. 4 were inverted, by fixing the source position to its known values. Afterwards, a model validation exercise was performed, inverting only for the source position, with large search intervals of $[1,10] \mathrm{km}$ and $[1,110] \mathrm{m}$ for range and depth, respectively. Only those environmental estimates conducive to a location estimate with an error of $5 \%$ of the search interval amplitude $-0.45 \mathrm{~km}$ in range, and $5.45 \mathrm{~min}$ depth - were considered hereafter, in both lower frequency (LF) and higher frequency (HF) bands. A set of 22 equi-spaced frequencies covering the whole band of each LFM signal was used for the inversion. To compute the sample correlation matrices, $N=10$ snapshots were considered. Thegeneticalgorithmsettings are summarizedin Table 1. The search bounds for the inverted parameters are shown in Table 2.
The inversion results for the water column and the bottom compressional speed are shown in Figs. 5 and 6, respectively. The estimated temperature profiles cannot be compared with ground truth values, since, for the acoustic transmission period, there are no available CTD measures. It can be seen that the surface temperature structure is the most difficult to estimate. This can be explained by the strong summer thermocline, which implies a low signal-to-noise ratio at the shallowest hydrophone depth. However, the surface estimates interval of 22.5 to $27.5^{\circ} \mathrm{C}$ agrees with the range 22.8 to $26.7{ }^{\circ} \mathrm{C}$ observed on days 16,17 and 19 of June, during the observation time in coincidence with the LF period. Regarding the inverted bottom parameters, it is observed that the compressional speed fairly agrees with the baseline model in Fig. 4. The other, not shown, inverted parameters values are quite variable, and oscillate significantly around the baseline values. This variability can be explained by a small sensitivity of the cost function to bottom environmental changes, since it considers data from only 3 hydrophones, or by a spacevariant bottom, different from that in the baseline model in Fig. 4, or, at last, by the equivalent acoustic model concept - leading to approximately equal acoustic

Table 2

Search bounds for the inverted parameters, for environmental assessment

\begin{tabular}{|c|c|c|c|}
\hline Model parameter & $\begin{array}{l}\text { Lower } \\
\text { bound }\end{array}$ & $\begin{array}{l}\text { Upper } \\
\text { bound }\end{array}$ & $\begin{array}{l}\text { Quantization } \\
\text { steps }\end{array}$ \\
\hline \multicolumn{4}{|l|}{ Water column } \\
\hline 1st EOF coefficient $\left({ }^{\circ} \mathrm{C}\right)$ & -15 & 15 & 128 \\
\hline 2nd EOF coefficient $\left({ }^{\circ} \mathrm{C}\right)$ & -7 & 7 & 128 \\
\hline \multicolumn{4}{|l|}{ Sediment } \\
\hline $\begin{array}{l}\text { Upper compressional } \\
\text { speed }(\mathrm{m} / \mathrm{s})\end{array}$ & 1470 & 1560 & 64 \\
\hline $\begin{array}{l}\text { Lower compressional } \\
\text { speed increment }(\mathrm{m} / \mathrm{s})\end{array}$ & 0 & 100 & 64 \\
\hline Density $\left(\mathrm{g} / \mathrm{cm}^{3}\right)$ & 1.2 & 2.5 & 64 \\
\hline $\begin{array}{l}\text { Compressional attenuation } \\
(\mathrm{dB} / \lambda)\end{array}$ & 0.01 & 0.5 & 32 \\
\hline Thickness (m) & 2 & 6 & 64 \\
\hline \multicolumn{4}{|l|}{ Subbottom } \\
\hline $\begin{array}{l}\text { Compressional speed } \\
\text { increment }(\mathrm{m} / \mathrm{s})\end{array}$ & 1 & 100 & 64 \\
\hline Density $\left(\mathrm{g} / \mathrm{cm}^{3}\right)$ & 1.2 & 2.5 & 16 \\
\hline $\begin{array}{l}\text { Compressional attenuation } \\
(\mathrm{dB} / \lambda)\end{array}$ & 0.01 & 0.5 & 16 \\
\hline \multicolumn{4}{|l|}{ Geometric } \\
\hline Lowest receiver depth (m) & 74.5 & 76.0 & 16 \\
\hline Tilt (rad) & -0.025 & 0.025 & 64 \\
\hline
\end{tabular}

The sediment lower compressional speed value is the increment relative to the estimated upper speed, and the subbottom compressional speed is the increment relative to the estimated sediment lower speed. 
(a) LF



(b) LF

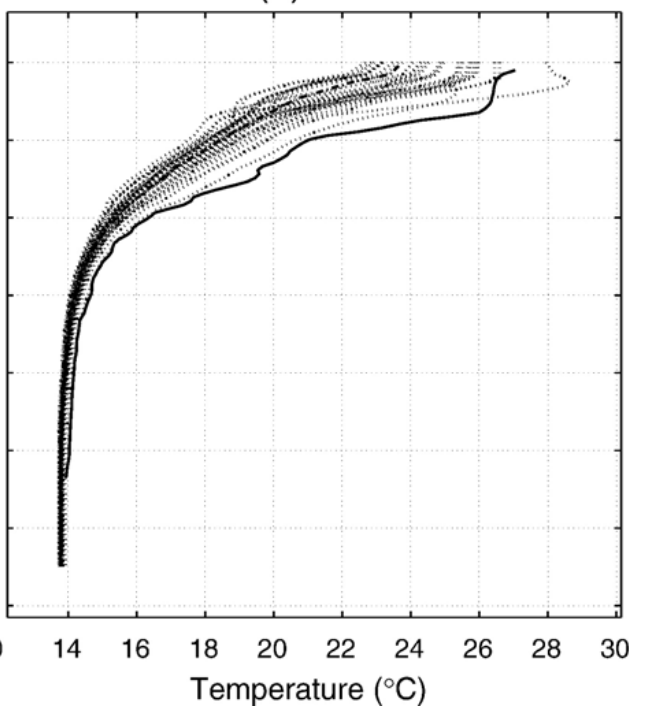

Fig. 5. Inversion results (dotted lines) for ocean temperature, in the two frequency bands 500-800 Hz (a) and 900-1200 Hz (b). The continuous lines represent the CTD measures closest in time to the acoustic experiment: before (a) and after (b) the experiment, at Julian times 171.34 and 171.76, respectively. The dash-dotted lines represent the inversion results closest in time to the above CTD measures, respectively.

responses corresponding to different combinations of bottom parameters.

\subsection{Acoustic assessment results}

In this paper, the concept illustrated in Fig. 1, to forecast the acoustic signal, was partially applied. At the end, the oceanographic forecast $\hat{\boldsymbol{\mu}}\left(t_{1}\right)$ as given by ocean circulation modelling, the environmental inversion results of water column and bottom parameters, and archival values of the bottom parameters, are available. This work intends to put in evidence the sensitivity of the output acoustic forecast $\hat{\mathbf{X}}\left(t_{1}\right)$ to different structures of the AO environment $\hat{\boldsymbol{\mu}}_{a}\left(t_{1}\right)$. The available environmental data coming from different sources (ocean prediction, acoustic inversion, etc.) can, in principle, be assimilated, in order to define an optimal vector $\hat{\boldsymbol{\mu}}_{a}\left(t_{1}\right)$ and compute the corresponding acoustic forecast. Here, a closed form for $\hat{\boldsymbol{\mu}}_{a}\left(t_{1}\right)$ is not derived, but the gain in utilizing multidisciplinary data is put in evidence. At the end, one can say that the inverted environment at present time contains valuable information to be combined with the predicted environment, in order to obtain optimal acoustic signal forecasts.

A set of 4 combinations of measured, forecast and inverted physical parameters was chosen, to define the acoustic model input $\hat{\boldsymbol{\mu}}_{a}\left(t_{1}\right)$, and then simulate the acoustic field along the ship-AOB1 track:

- Water column forecast and historical bottom data: in this combination, the AO environment is defined by linearly-interpolated averaged MODAS-NCOM predicted temperature profiles for each geometric source-receiver slice, and archival bottom parameters, taken from (Gingras, 1994). The considered predicted temperature profiles are contained in the interval shown in Fig. 3(a), and the bottom parameters are given in Fig. 4.

- Water column forecast and inverted bottom profile: here, the AO environment is defined by the MODAS-NCOM predicted values for the water column temperature, and the inverted bottom parameters mentioned in Section 3.2.

- Inverted water column and bottom parameters: the AO environment is defined by the inverted temperature profiles and bottom parameters, mentioned in Section 3.2.

- Inverted water column and historical bottom parameters: here, the AO environment is defined by the inverted temperature profiles in Fig. 5, and the bottom archival parameters in Fig. 4.

In predicting the acoustic field, it is necessary first to define what features of the field are to be estimated, and a functional which quantifies the proximity between the data and the model. For this purpose, one can resort to a multitude of cost functions. For example, they may involve either time or frequency characteristics of the signals, and depend on cross-correlations or differences between the data and the acoustic model output. Among a possibly infinite number of cost functions that can be defined, it is guaranteed, at least in simulated noiseless 

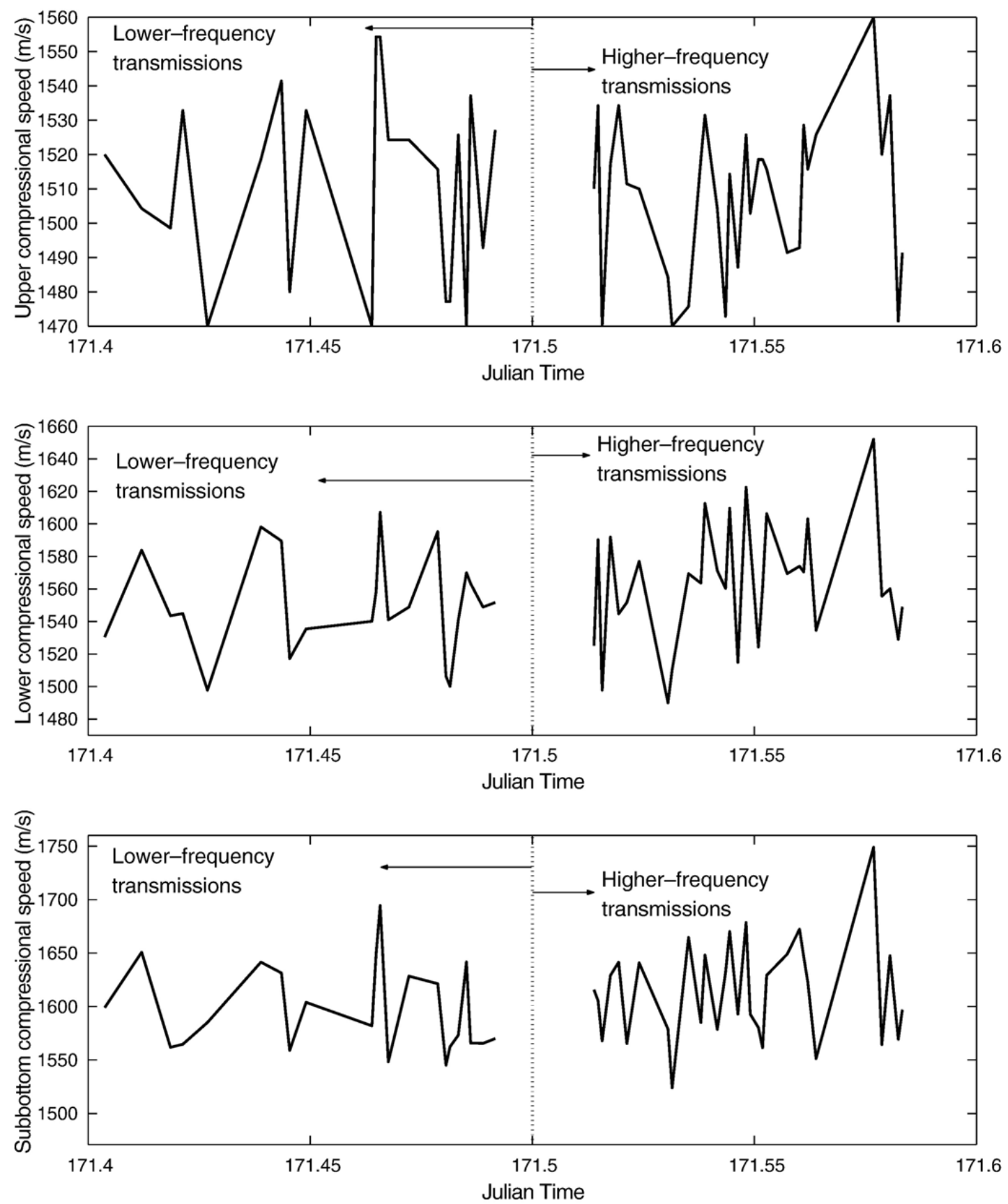

Fig. 6. Inversion results for the compressional speed of the sediment and subbottom layers, in the lower and higher frequency bands.

data, that the global optimum of any cost function will be attained at the $\mathrm{AO}$ environment that generated the acoustic data. Outside the global optimum, each cost function may have a different variational behaviour. This topic is supported by the illustration in Fig. 7, where two cost functions are shown, for simulated noiseless data generated with the baseline model in Fig. 4. Here, the reference data was generated with a null value for the first EOF coefficient $\alpha_{1}$. By generating acoustic replicas with a variable $\alpha_{1}$, both the Bartlett- based cost function (6) and the transmission loss (TL)based cost function

$P_{\mathrm{TL}}(\boldsymbol{\mu})=1-\frac{\left\|\mathbf{T L}_{Y}-\mathbf{T L}_{H}(\boldsymbol{\mu})\right\|}{2}$,

where $\mathbf{T L}_{Y}\left(\mathbf{T L}_{H}(\boldsymbol{\mu})\right.$, respectively) are extended normalized versions of vectors containing the data (replica, respectively) TL at all frequencies and sensors, are plotted. In the figure, it is seen that the overall 


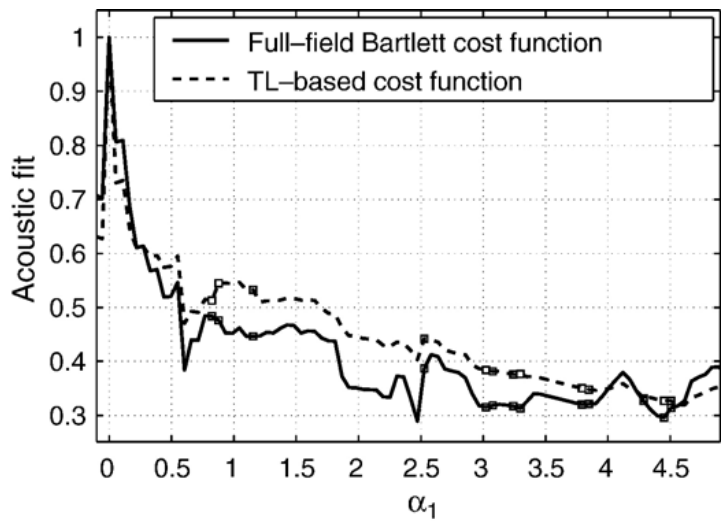

Fig. 7. Two possible cost functions in the coefficient of the first empirical orthogonal function of temperature $-\alpha_{1}$ - with simulated noiseless data: Bartlett power (6) (-) and transmission loss-based cost function (7) (- - ). The reference data was generated with the baseline model in Fig. 4, and a null value for the EOF coefficient. The squares indicate the points where the functions derivatives have opposite sign.

behaviour of both cost functions is similar. Also, in some cases, a higher acoustic match in the TL sense does not imply a higher match in the Bartlett sense see the opposite functional variations, in the particular points marked by squares, in Fig. 7. This can be explained by two reasons. First, the cost functions involve different quantities: the TL-based function compares the magnitude of the acoustic field, while the Bartlett function compares the full field, in amplitude and phase. Second, both cost functions synthesize the acoustic match at a set of spatial positions and frequencies in a single value. Lower matches at some points can be obscured by the integral cost value and may not coincide between the different cost functions. Thus, in Fig. 7, if $\alpha_{1}$ approaches 1 by the left side, the environmental match is decreased, the acoustic match in the Bartlettsense is decreased, but the acoustic match in the TLsense is increased. The opposite effect can be observed when $\alpha_{1}$ approaches 3 by the right side. These subtle differences between the environmental mismatch implications on different cost functions are enlarged when considering actual noisy data.

To assure consistent results and conclusions, in the problem at hand, it was chosen to quantify the acoustic prediction quality by the same functional used in the inversion process. The acoustic simulation outputs corresponding to the four environmental parameter vectors defined as previously explained, were compared to actual data, using Eq. (6), and this comparison is illustrated in Fig. 8. In both plots, as expected, it can be seen that the 'acoustic forecast' corresponding to the inverted physical vector (dashed line) is closest, in the sense of Eq. (6), to the measured data. From the curves representing mixed heterogeneous environments, the one corresponding to the inverted temperature (continuous line) is generally

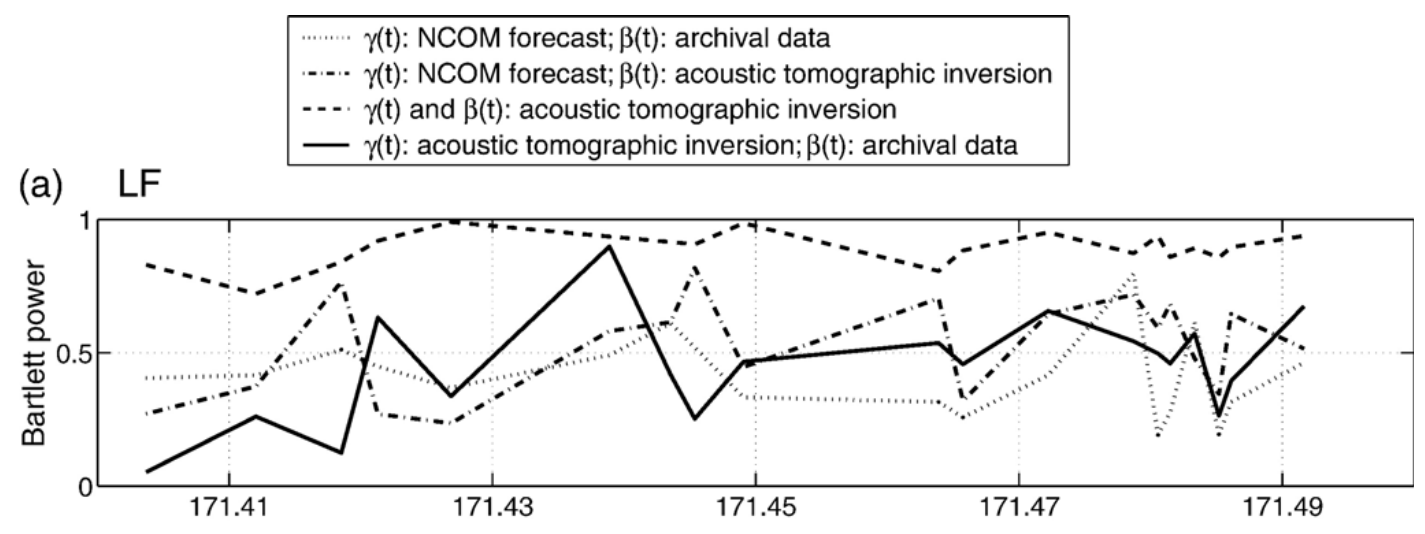

(b) $\mathrm{HF}$



Fig. 8. Acoustic forecast results: Bartlett power (6), for the different combinations of water column and bottom parameters, $\boldsymbol{\gamma}\left(t_{1}\right)$ and $\boldsymbol{\beta}\left(t_{1}\right)$, respectively, taken from inverted, archival and forecast acoustic and/or oceanographic quantities, for the LF (a) and HF (b) periods. The power was calculated for the acoustic inversion times. 
larger than the other curves. The curve which corresponds to the water column forecast and the bottom archival data assumes generally the smallest values, specially in the lower frequency period. In the initial part of the LF period, the higher values can be explained by the large source depth of $\approx 105 \mathrm{~m}$, which makes the transmission loss structure more insensitive to the differences between the thermocline depths of the inverted and predicted profiles - compare Figs. 5 and 3, respectively. These facts support the idea that, even in shallow water, both the water column structure and the geo-acoustic play a significant role on the definition of the acoustic signals. From all the curves in Fig. 8, the periods where the continuous line has the second largest values indicate a predominance of the water column properties in the definition of the acoustics; the periods where the second largest values are given by the dash-dotted curve indicate the predominance of an optimum definition of the geo-acoustic properties.

The results reinforce the idea that, assuming a given environmental parameterization structure (range-dependent or -independent water depth, temperature or other parameters, number of acoustic layers, etc.), comprising both oceanographic and non-oceanographic parameters, if the purpose is to give as output an accurate acoustic signal (here, to compute an 'acoustic forecast' outcome), then, the process of acoustic inversion plays a significant role in determining adequate values for the acoustic model input. Should the model input structure be slightly modified (e.g. increasing the detail of the bathymetry, decreasing the complexity of the bottom parameterization), then, in principle, it would be possible to obtain other 'acoustic forecast' outcomes with the same accuracy, by re-adjusting the values for the parameters of the new environmental model.

Let us refer back to Figs. 5 and 6. It can be seen, by comparing the reasonably stable Bartlett values in the dashed line of Fig. 8 with the unstable values for the water column - Fig. 5 - and some of the bottom parameter estimates - Fig. 6 - that the unstability observed in the inverted values can be attributed to the equivalent acoustic model concept. Considering the higher frequency period - see Fig. 2 - where the bathymetry is mildly range-independent, it is unlikely that the referred physical parameters have such significant different values. To attain the objective of acoustic forecast, it is desirable to perform acoustic simulations with stable environmental parameters, in order to maximize the forecast window duration and minimize the forecast uncertainty. This is possible, in principle, by combining the inversion results in Fig. $6-$ for assumed space-invariant parameters - in order to obtain a single environmental parameter set which contains most of the acoustic information contained in any of the outcomes in Fig. 6.

In conclusion, having at hand only the water column conditions forecast and archival bottom data from a given area of interest, is seen to not provide enough information to obtain an optimum acoustic output, where optimality must be defined e.g. in the Bartlett sense, TL-sense, etc. This is a natural consequence of model-based acoustic prediction, and also of dealing with estimated quantities with intrinsic variances: the water column conditions forecast is the output of an oceanographic estimator with a coarse resolution in space, and optimizing only for oceanographic quantities; the archival bottom data, taken either from measures or from 'historical' acoustic inversions, also contains uncertainties, and usually correspond to an even coarser space resolution.

\section{Conclusion}

This paper considered the problem of environmental and acoustic assessment with data from the MREA '03 sea trial, an acoustic-oceanographic experiment where the acoustic signals of interest were received in a 3hydrophone array, which is part of the self-contained Acoustic-Oceanographic Buoy. Environmental assessment is an established discipline, where ocean prediction systems, and more recently, acoustic inversion tools, play a fundamental role. This paper has pointed out the importance of combining such estimation tools, to obtain what was designated by acoustic assessment, i.e., the estimation of the present and future acoustic field on the observing array.

The problem of predicting the acoustic field on the array was conceptually approached. Here, the focus was on the strong dependence of the acoustic forecast result on the acoustic model parameterization. This allowed to formulate the problem of acoustic forecast as a problem of estimating the optimal acoustic model environmental input. With the aim of predicting the acoustic signal, a comparison was done between actual acoustic data and candidate simulations. The latter differed on the structure of the environmental input, which alternatively considered water column conditions coming from forecasts or acoustic inversion, and geo-acoustic properties derived from archival data or acoustic inversion. The results have shown that the acoustic simulated field closest to the actual field is the one corresponding to the inverted environment. This shows the gain that can be obtained from acoustic inversion, by comparison to traditional acoustic forecast techniques, where the 
forecast water temperature and geo-acoustic properties (even if coming from direct core measurements) are fed directly to the acoustic propagation model. Acoustic inversion can be interpreted as a 'fine-tuner' of the propagation model input parameters.

Considering the present study, the intrinsic problem is that, at the time of the forecast estimation, there is no information about the signal received in the future time, and the possibility of doing acoustic inversion is confined to acoustic data that might be acquired at present time. In this paper, the 'acoustic forecast' results quality are to be treated as an upper bound, since the environmental parameter considered to compute the acoustic field was determined by acoustic inversion of the offline future data.

Future work will use training data sets consisting of environmental inversion results and environmental nowcasts, to estimate transforming functionals between these two environment outcomes. The transforming functionals are to be used with water column forecasts, to produce estimates of future inverted environmental outcomes, and finally future acoustic outputs (acoustic forecasts) on the array.

\section{Acknowledgments}

The authors thank FCT, for funding, under programs POSI and POCTI, scholarship no. SFRH/BD/9032/2002 and NUACE project, no. POSI/CPS/47824/2002, and all the people involved in the AOB-JRP, with especial thanks to the scientist-in-charge Emanuel FerreiraCoelho, for conducting the MREA '03 sea trial. The reviewers' comments were quite valuable regarding the paper direction and physical considerations.

\section{References}

Ferla, C., Porter, M., Jensen, F., 1993. C-SNAP: Coupled SACLANTCEN normal mode propagation loss model. Memorandum SM274. SACLANTCEN Undersea Research Center, La Spezia, Italy.
Gingras, D., 1994. North Elba sea trial summary, Tech. Rep. Aug., report and acoustic dataset are published at the Signal Processing Information Base at Rice University on the world wide web. NATO SACLANTCEN, La Spezia. http://spib.rice.edu.

Jesus, S.M., Soares, C., Silva, A.J., 2003. Acoustic Oceanographic Buoy testing during the Maritime Rapid Environmental Assessment 2003 sea trial. Report, vol. 04/03. SiPLAB, University of Algarve, Faro.

Jesus, S.M., Soares, C., Coelho, E., Picco, P., 2006. An experimental demonstration of blind ocean acoustic tomography. J. Acoust. Soc. Am. 119 (3), 1420-1431 (March).

Lermusiaux, P.F.J., Chiu, C.-S., 2002. Four-dimensional data assimilation for coupled physical-acoustical fields. Proceedings of the “Acoustic Variability, 2002" conference in Lerici, Italy, pp. 417-424.

Martin, P., 2000. Description of the Navy Coastal Ocean Model version 1.0, report NRL/FR/7322-00-9962. Naval Research Laboratory.

Robinson, A.R., Lermusiaux, P.F.J., 2003. Prediction systems with data assimilation for coupled ocean science and ocean acoustics. Sixth International Conference on Theoretical and Computational Acoustics (ICTCA), Honolulu, HI, 11 August, 2003, pp. 325-342.

Robinson, A.R., Sellschopp, J., 2002. Rapid assessment of the coastal ocean environment. In: Pinardi, N., Woods, J.D. (Eds.), Ocean Forecasting: Conceptual Basis and Applications. Springer, pp. 203-232.

Robinson, A.R., Abbot, P., Lermusiaux, P.F.J., Dillman, L., 2002. Transfer of uncertainties through physical-acoustical-sonar endto-end systems: a conceptual basis. In: Pace, N.G., Jensen, F.B. (Eds.), Acoustic Variability. SACLANTCEN, pp. 603-610.

Soares, C., Jesus, S., 2003. Broadband matched-field processing: coherent and incoherent approaches. J. Acoust. Soc. Am. 113 (5), 2587-2598 (May).

Soares, C., Jesus, S., Silva, A., Coelho, E., 2004. Acoustic Oceanographic Buoy testing during the Maritime Rapid Environmental Assessment 2003 sea trial. Proceedings of ECUA'04. Delft, The Netherlands, pp. 271-279 (July). 